J. Chang

Nagoya Math. J.

Vol. 195 (2009), 77-95

\title{
NORMALITY, QUASINORMALITY AND PERIODIC POINTS
}

\author{
JIANMING CHANG
}

\begin{abstract}
Let $M \geq 1$ be a positive number. Let $\mathcal{F}$ be a family of holomorphic functions $f$ in some domain $D \subset \mathbb{C}$ for which there exists an integer $k=k(f) \geq$ 2 such that $\left|\left(f^{k}\right)^{\prime}(\zeta)\right| \leq M^{k}$ for every periodic point $\zeta$ of period $k$ of $f$ in $D$. We show first that $\mathcal{F}$ is quasinormal of order at most one in $D$. This strengthens a result of W. Bergweiler. Secondly, for the case $M=1$, we prove that $\mathcal{F}$ is normal in $D$ if there exists a positive number $K<3$ such that $\left|f^{\prime}(\eta)\right| \leq K$ for each $f \in \mathcal{F}$ and every fixed point $\eta$ of $f$ in $D$. This improves a result of M. Essén and S. J. Wu. We also construct an example which shows that the condition $\left|f^{\prime}(\eta)\right| \leq K<3$ can not be replaced by $\left|f^{\prime}(\eta)\right|<3$.
\end{abstract}

\section{$\S 1$. Introduction and main results}

Both the concepts of normal families and quasinormal families were introduced by Montel. Let $D \subset \mathbb{C}$ be a domain and let $\mathcal{F}$ be a family of holomorphic functions in $D$. Then

(i) $\mathcal{F}$ is said to be normal in $D$ in the sense of Montel if each sequence $\left\{f_{n}\right\} \subset \mathcal{F}$ contains a subsequence which converges locally uniformly in $D$ to a holomorphic function or diverges locally uniformly in $D$ to $\infty$. See [15], [19], [21].

(ii) $\mathcal{F}$ is said to be quasinormal in $D$ in the sense of Montel if for every sequence $\left\{f_{n}\right\} \subset \mathcal{F}$ there exists a subsequence $\left\{f_{n_{j}}\right\}$ and a finite set $E \subset D$ such that $\left\{f_{n_{j}}\right\}$ converges locally uniformly in $D \backslash E$ to a holomorphic function or $\infty$. Furthermore, if there exists a smallest integer $q$ such that the cardinality $|E| \leq q$ for all exceptional sets $E$, then $\mathcal{F}$ is called quasinormal of order $q$. See [19].

Received January 15, 2007.

Revised August 23, 2008, January 16, 2009.

Accepted January 19, 2009.

2000 Mathematics Subject Classification: 30D45, 30D05, 37F10, 37C25.

Supported by the NNSF of China (Grant Nos. 10671093 and 10871094), the NSFU of Jiangsu, China (Grant No. 08KJB110001), the Qing Lan Project of Jiangsu, China and the SRF for ROCS, SEM. 
Thus a normal family is quasinormal of order 0 . In recent years, there have been many interesting results on (quasi)normal families that concern the existence of periodic points.

Let $D \subset \mathbb{C}$ be a domain and let $f: D \rightarrow \mathbb{C}$ be a holomorphic function. Then the iterates $f^{n}: D_{n} \rightarrow \mathbb{C}$ of $f$ are defined inductively by $D_{1}=D$, $f^{1}=f$ and $D_{n}=f^{-1}\left(D_{n-1}\right)=\left\{z \in D: f(z) \in D_{n-1}\right\}, f^{n}=f^{n-1} \circ f$ for $n \geq 2$. Note that $D_{n+1} \subset D_{n} \subset D$ for all $n \in \mathbb{N}$. See [3], [7], [8], [11], [13], [14].

Let $z_{0} \in D$. If there exists a positive integer $p \in \mathbb{N}$ such that $z_{0} \in$ $D_{p}, f^{p}\left(z_{0}\right)=z_{0}$ and $f^{j}\left(z_{0}\right) \neq z_{0}$ for all $1 \leq j \leq p-1$, then $z_{0}$ is said to be a periodic point of $f$ of period $p$, and the corresponding cycle $\left\{z_{0}, f\left(z_{0}\right), \ldots, f^{p-1}\left(z_{0}\right)\right\} \subset D$ is said to be a periodic cycle of period $p$. A periodic point with period 1 is said to be a fixed point. Define the multiplier of the periodic point $z_{0}$ (and the corresponding cycle) by $\lambda=\left(f^{p}\right)^{\prime}\left(z_{0}\right)$. According to $|\lambda|<1,|\lambda|=1$, or $|\lambda|>1$, the periodic point $z_{0}$ (and the corresponding cycle) is said to be attracting, neutral, or repelling. See [9]. We also call a fixed point weakly repelling if it is repelling or has multiplier 1.

In complex dynamics, periodic points play an important role. For example, the Julia set of a meromorphic function is the closure of the set of its repelling periodic points. See [9]. Thus it is well worth studying normal or quasinormal families of holomorphic functions defined by conditions on fixed points or periodic points.

M. Essén and S. J. Wu [13], [14] proved the following result, thereby answering affirmatively a question of Yang [20, Problem 8].

Theorem A. ([13], [14]) Let $\mathcal{F}$ be a family of functions holomorphic in $D \subset \mathbb{C}$. If for every $f \in \mathcal{F}$, there exists an integer $k=k(f) \geq 2$ such that $f^{k}$ has no repelling fixed point in $D$, then $\mathcal{F}$ is normal in $D$.

W. Bergweiler [7], improving a result due to D. Bargmann and W. Bergweiler [3], considered an analogue under a weaker condition than that of Theorem A, and proved

Theorem B. ([7]) Let $M \geq 1$ be a positive number and let $\mathcal{F}$ be a family of functions holomorphic in $D \subset \mathbb{C}$. If for every $f \in \mathcal{F}$, there exists an integer $k=k(f) \geq 2$ such that $\left|\left(f^{k}\right)^{\prime}(\zeta)\right| \leq M$ for all periodic points $\zeta$ of period $k$ of $f$ in $D$, then $\mathcal{F}$ is quasinormal of order at most 1 in $D$. 
In the present paper, we will improve both Theorem A and Theorem B. Following the idea of Bergweiler [7] with some additional attention to the multipliers, we have the following result.

THEOREM 1. Let $M \geq 1$ be a positive number, $D \subset \mathbb{C}$ be a domain, and $\mathcal{F}$ be a family of functions holomorphic in $D$. If for every $f \in \mathcal{F}$, there exists an integer $k=k(f) \geq 2$ such that $\left|\left(f^{k}\right)^{\prime}(\zeta)\right| \leq M^{k}$ for all periodic points $\zeta$ of period $k$ of $f$ in $D$, then $\mathcal{F}$ is quasinormal of order at most 1 in $D$.

Theorem 1 strengthens Theorem B, since $\left\{M^{k(f)}\right\}$ may be unbounded. From the family $\{n z: n \in \mathbb{N}\}$, we see that, to obtain normality, some further assumptions are needed. In this direction, we have

TheOREm 2. Let $M \geq 1$ be a positive number, $D \subset \mathbb{C}$ be a domain, and $\mathcal{F}$ be a family of functions holomorphic in $D$. If for every $f \in \mathcal{F}, f$ has no weakly repelling fixed point in $D$, and there exists an integer $k=k(f) \geq 2$ such that $\left|\left(f^{k}\right)^{\prime}(\zeta)\right| \leq M^{k}$ for all periodic points $\zeta$ of period $k$ of $f$ in $D$, then $\mathcal{F}$ is normal in $D$.

The following example shows that the condition that every $f \in \mathcal{F}$ has no weakly repelling fixed point can not be replaced by the condition that every $f \in \mathcal{F}$ has no repelling fixed point in $D$.

EXAMPLE 1. Let

$$
\mathcal{F}=\left\{f_{n}(z)=z+n z^{2}: n \in \mathbb{N}\right\} .
$$

Then $\mathcal{F}$ is a family of functions holomorphic in $\mathbb{C}$. Every $f_{n} \in \mathcal{F}$ has a single fixed point $z=0$ in $\mathbb{C}$ with multiplier 1 , so that has no repelling fixed point in $\mathbb{C}$. And for every $f_{n} \in \mathcal{F}$, we have

$$
f_{n}^{2}(z)=z+n z^{2}\left(n^{2} z^{2}+2 n z+2\right)
$$

so that $\left|\left(f_{n}^{2}\right)^{\prime}(\zeta)\right| \leq(\sqrt{5})^{2}$ for all periodic points $\zeta$ of period 2 of $f_{n}$ in $\mathbb{C}$. But $\mathcal{F}$ is not normal in $\mathbb{C}$ since $f_{n}(0)=0$ and $f_{n}(1 / \sqrt{n})=1+1 / \sqrt{n} \rightarrow 1$.

However, it does not seem unlikely that if $M<\sqrt{5}$ in Theorem 2, then such a replacement is permissible. Here we consider the special case that $M=1$ and prove the following result, which is a generalization of Theorem A. 
TheOREM 3. Let $K<\infty$ be a positive number, $D \subset \mathbb{C}$ be a domain, and $\mathcal{F}$ be a family of functions holomorphic in $D$. If for every $f \in \mathcal{F}$, $\left|(f)^{\prime}(\eta)\right| \leq K$ for every fixed point $\eta$ of $f$ in $D$, and there exists a positive integer $k=k(f)$ such that $f$ has no repelling periodic points of period $k$ in $D$, then $\mathcal{F}$ is normal in $D$, provided that one of the following conditions holds:

(a) $K<3$ and $k(f) \geq 2$ for all $f \in \mathcal{F}$;

(b) $K<2 \sqrt{2}+1$ and $k(f) \geq 3$ for all $f \in \mathcal{F}$;

(c) $K<\infty$ and $k(f) \geq 4$ for all $f \in \mathcal{F}$.

The simple example $\{n z\}$ shows that it is necessary to assume that the multipliers of the fixed points of $f$ for all $f \in \mathcal{F}$ are uniformly bounded. We also construct two examples in Section 4 which show that $\left|(f)^{\prime}(\eta)\right| \leq K<3$ and $\left|(f)^{\prime}(\eta)\right| \leq K<2 \sqrt{2}+1$ can not be replaced by $\left|(f)^{\prime}(\eta)\right|<3$ and $\left|(f)^{\prime}(\eta)\right|<2 \sqrt{2}+1$ respectively, so that the conditions in Theorem 3 are sharp.

The plan of this paper is as follows. In Section 2, we state the required preliminary results, including the Ahlfors islands theorem, the fundamental theorem of polynomial-like mappings and Zalcman-Pang's rescaling lemma. In Section 3, we give the proofs of our results. In Section 4, we construct the two examples mentioned above, and finally in Section 5, we give a remark about the family of meromorphic functions.

\section{§2. Notation and preliminary results}

For $z_{0} \in \mathbb{C}$ and $r>0, D\left(z_{0}, r\right)=\left\{z \in \mathbb{C}:\left|z-z_{0}\right|<r\right\}$ and $\bar{D}\left(z_{0}, r\right)=$ $\left\{z \in \mathbb{C}:\left|z-z_{0}\right| \leq r\right\}$.

For two functions $f$ and $g$, we say they are similar, if there exist constants $a(\neq 0), b$ such that $f(z)=\phi^{-1} \circ g \circ \phi(z)$, where $\phi(z)=a z+b$. See [1].

To prove the theorems, we require some preliminary results. One of the central tools is the Ahlfors islands theorem coming from Ahlfors theory of covering surfaces. The idea of applying the Ahlfors islands theorem to study normal or quasinormal families of holomorphic functions defined by conditions on fixed points or periodic points is due to Essén-Wu [13], [14] and Bargmann-Bergweiler [3].

Let $f: D \rightarrow \mathbb{C}$ be a holomorphic function. For a given Jordan domain $\Omega \subset \mathbb{C}$, a connected component $\Omega_{0}$ of $f^{-1}(\Omega)$ is called a simple island over $\Omega$ if $\left.f\right|_{\Omega_{0}}: \Omega_{0} \rightarrow \Omega$ is a conformal homeomorphism. 
The Ahlfors islands theorem is well-known and plays an important role in complex dynamics. For example, using the Ahlfors islands theorem, Baker [2] proved that the Julia set of a transcendental entire function is the closure of the set of its repelling periodic points. The following version of the Ahlfors islands theorem can be found in [6].

Lemma 1. ([6, Theorem A.1]) Let $D \subset \mathbb{C}$ be a domain and let $D_{1}, D_{2}$, $D_{3}$ be Jordan domains in $\mathbb{C}$ whose closures are pairwise disjoint. Then the family of all holomorphic functions $f: D \rightarrow \mathbb{C}$ which have no simple island over any $D_{j}$ is normal in $D$.

The second central tool comes from the theory of polynomial-like mappings of Douady and Hubbard [12]. This theory explains how the understanding of polynomials is not only interesting per se, but helps understand a wider class of functions that locally behave as polynomials do. We say $(f, U, V)$ is a polynomial-like mapping of degree $d$ if $U, V \subset \mathbb{C}$ are bounded and simply connected domains with $\bar{U} \subset V$ and $f: U \rightarrow V$ is a proper map of degree $d$. We also call a polynomial-like mapping of degree 1 a regular map, cf. [7]. Obviously, a regular map is a holomorphic and bijective map. The following well-known result which is called Straightening Theorem explains the relation between polynomial-like mappings and actual polynomials.

Lemma 2. ([12, Theorem 1], [9, Theorem VI.1.1], cf. [3, Lemma 2]) Let $(f, U, V)$ be a polynomial-like mapping of degree $d \geq 2$. Then there exists a polynomial $P$ of degree $d$ and a quasi-conformal map $\phi: \mathbb{C} \rightarrow \mathbb{C}$ with $\phi(z)=z+o(1)$ near $\infty$ such that $f(z)=\phi^{-1} \circ P \circ \phi(z)$ for all $z \in U$. Moreover, $\phi(U)$ contains the filled-in Julia set $K(P)$ of $P$, and hence all periodic points of $P$.

We also require some useful facts about polynomial-like mappings.

Lemma 3. ([14, Lemma 3]) Let $(f, U, V)$ be a polynomial-like mapping of degree $d \geq 2$. Then $f$ has at least one weakly repelling fixed point in $U$.

LEMma 4. ([7, Lemma 2.2]) Let $(f, U, V)$ be a polynomial-like mapping of degree $d \geq 2$, and $D_{1}, D_{2}$ be two Jordan domains with pairwise disjoint closures contained in $V$. Then there exist two domains $U_{1}, U_{2} \subset U$, each of which is a simple island over $D_{1}$ or $D_{2}$. 
Lemma 5. Let $U \subset D(c, r) \subset D(a, \varepsilon)$ be a simply-connected domain. Let $f: U \rightarrow D(a, \lambda \varepsilon),(\lambda \geq 2)$ be a regular map. Then $f$ has a fixed point $z_{0} \in U$ which satisfies

$$
\left|f^{\prime}\left(z_{0}\right)\right| \geq \frac{3 \lambda \varepsilon}{4 r}
$$

Proof. Without loss of generality, assume that $a=0$ and $\lambda \varepsilon=1$. It follows from Rouché's theorem that $f$ has a fixed point $z_{0} \in U$ and then $\left|z_{0}\right|<\varepsilon=1 / \lambda \leq 1 / 2$. Let $\phi(z)=\left(f^{-1}(z)-c\right) / r$. Then, $\phi$ is a self-mapping of the unit disk and $\phi\left(z_{0}\right)=\left(z_{0}-c\right) / r$. Applying the Schwarz-Pick lemma to $\phi$, we have

$$
\left|\phi^{\prime}\left(z_{0}\right)\right| \leq \frac{\left|\phi^{\prime}\left(z_{0}\right)\right|}{1-\frac{\left|z_{0}-c\right|^{2}}{r^{2}}} \leq \frac{1}{1-\left|z_{0}\right|^{2}},
$$

and, since $\phi^{\prime}\left(z_{0}\right)=1 / r f^{\prime}\left(z_{0}\right)$,

$$
\left|f^{\prime}\left(z_{0}\right)\right| \geq \frac{1}{r}\left(1-\left|z_{0}\right|^{2}\right) \geq \frac{3}{4 r}
$$

This proves Lemma 5.

Lemma 6. Let $U \subset D(a, \varepsilon)$ and $V \supset D(b, \lambda \varepsilon),(\lambda \geq 2)$ be simplyconnected domains. Let $f: U \rightarrow V$ be a regular map. Then for $D(c, r) \subset$ $D(b, \varepsilon)$, there exists a disk $D\left(c^{\prime}, r^{\prime}\right) \subset D(a, \varepsilon)$ such that $r^{\prime}<3 r / \lambda$ and $f^{-1}(D(c, r)) \subset D\left(c^{\prime}, r^{\prime}\right)$.

Proof. Without loss of generality, assume that $a=b=0$ and $\lambda \varepsilon=1$. Let $\phi(z)=f^{-1}(z) / \varepsilon$, which is a self-mapping of the unit disk. For $\zeta \in$ $D(c, r)$, by the Schwarz-Pick lemma, we have

$$
\frac{|\phi(\zeta)-\phi(c)|}{|1-\overline{\phi(\zeta)} \phi(c)|} \leq \frac{|\zeta-c|}{|1-\bar{\zeta} c|}
$$

Since

$$
\begin{gathered}
\frac{|\zeta-c|}{|1-\bar{\zeta} c|} \leq \frac{r}{1-1 / \lambda^{2}} \text { and } \\
\frac{|\phi(\zeta)-\phi(c)|}{|1-\overline{\phi(\zeta)} \phi(c)|} \geq \frac{1}{2}|\phi(\zeta)-\phi(c)|=\frac{1}{2 \varepsilon}\left|f^{-1}(\zeta)-f^{-1}(c)\right|
\end{gathered}
$$

we obtain

$$
\left|f^{-1}(\zeta)-f^{-1}(c)\right| \leq \frac{2 \varepsilon r}{1-1 / \lambda^{2}}<\frac{3 r}{\lambda}
$$

Lemma 6 is proved. 
We also require the following results which concern with the existence of (repelling) periodic points of given periods. It was Baker [1] who first studied this problem. His conjecture [16, Problem 2.20] about this question for transcendental entire functions has been confirmed by Bergweiler [5].

LEMma 7. ([5, Theorem 1]) Let $f$ be a transcendental entire function and $k \geq 2$ be an integer. Then $f$ has infinitely many repelling periodic points of period $k$.

Lemma 8. ([10, Theorem 1], cf. [4, Satz 1]) Let $P$ be a polynomial of degree $d \geq 2$, and $k \geq 2$ be an integer. If $P$ has no repelling periodic cycle of period $k$, then $k \in\{2,3\}$ and $P$ is similar to one of the following polynomials: for $k=2$,

(a) $Q(z)=z^{2}+c$ with constants $c$ satisfying $|c+1| \leq 1 / 4$,

(b) $Q(z)=z^{3}-\left(2+3 c^{2}\right) z+2 c^{3}$ with constants c satisfying $\left|c^{2}-1 / 36\right| \leq$ $1 / 36$, and

(c) $Q(z)=z^{4}+(-1 \pm 2 i) z$;

while for $k=3$,

(d) $Q(z)=z^{2}-7 / 4$.

As a corollary, we get

Lemma 9. Let $P$ be a polynomial of degree $d \geq 2$, and $k \geq 2$ be an integer such that $P$ has no repelling periodic cycle of period $k$. Then $k \in$ $\{2,3\}$ and

$$
\max _{P(z)=z}\left\{\left|P^{\prime}(z)\right|\right\} \geq \begin{cases}2 \sqrt{2}+1, & \text { if } k=3 \\ 3, & \text { if } k=2 .\end{cases}
$$

Proof. By Lemma 8, we have $k \in\{2,3\}$.

First suppose $k=3$. Then by Lemma $8, P$ is similar to the polynomial $Q(z)=z^{2}-7 / 4$. As for two similar polynomials $P$ and $Q$, we have

$$
\max _{P(z)=z}\left\{\left|P^{\prime}(z)\right|\right\}=\max _{Q(z)=z}\left\{\left|Q^{\prime}(z)\right|\right\}
$$

so we may assume $P(z)=z^{2}-7 / 4$. Clearly, we have

$$
\max _{P(z)=z}\left\{\left|P^{\prime}(z)\right|\right\}=2 \sqrt{2}+1 .
$$


This proves the case $k=3$.

Now suppose $k=2$. Then as above, we may assume $P(z)=z^{2}+c$ for some $c$ satisfying $|c+1| \leq 1 / 4$ or $P(z)=z^{3}-\left(2+3 c^{2}\right) z+2 c^{3}$ for some $c$ satisfying $\left|c^{2}-1 / 36\right| \leq 1 / 36$ or $P(z)=z^{4}+(-1 \pm 2 i) z$.

If $P(z)=P_{c}(z)=z^{2}+c$ for some $c$ satisfying $|c+1| \leq 1 / 4$. Choose a constant $t$ such that $\operatorname{Re}(t) \geq 0$ and $c=\left(1-t^{2}\right) / 4$. Then $P(z)=z^{2}+(1-$ $\left.t^{2}\right) / 4=z+\left(z-\frac{1+t}{2}\right)\left(z-\frac{1-t}{2}\right)$ and $\left|t^{2}-5\right| \leq 1$. Since $\operatorname{Re}(t) \geq 0$, we see that $\operatorname{Re}(t) \geq 2$. Thus we see that

$$
\max _{P(z)=z}\left\{\left|P^{\prime}(z)\right|\right\}=\max \{|1+t|,|1-t|\} \geq 3
$$

Next suppose $P(z)=P_{c}(z)=z^{3}-\left(2+3 c^{2}\right) z+2 c^{3}$ for some $c$ satisfying $\left|c^{2}-1 / 36\right| \leq 1 / 36$. Note

$$
\max _{P_{0}(z)=z}\left\{\left|P_{0}^{\prime}(z)\right|\right\}=7 .
$$

For $c \neq 0$, we claim $P_{c}$ has three different fixed points with different multipliers. For otherwise, suppose $P_{c}\left(z_{1}\right)=z_{1} \neq z_{2}=P_{c}\left(z_{2}\right)$ and $P_{c}^{\prime}\left(z_{1}\right)=$ $P_{c}^{\prime}\left(z_{2}\right)$. As $P_{c}^{\prime}(z)=3 z^{2}-\left(2+3 c^{2}\right)$, we get $z_{1}^{2}=z_{2}^{2}$ so that $z_{1}=-z_{2}$. It with $P_{c}\left(z_{1}\right)=z_{1}$ and $z_{2}=P_{c}\left(z_{2}\right)$ shows that $c=0$. A contradiction. Moreover the three different multipliers are exactly three roots of the equation

$$
\lambda^{3}-\left(12+9 c^{2}\right) \lambda^{2}+\left(21+18 c^{2}\right) \lambda+98+315 c^{2}+324 c^{4}=0 .
$$

In fact, let $z=z_{0}$ be a fixed point of $P_{c}$, and let $\lambda=P_{c}^{\prime}\left(z_{0}\right)$. Then $z_{0}^{3}-\left(3+3 c^{2}\right) z_{0}+2 c^{3}=0$ and $\lambda=3 z_{0}^{2}-\left(2+3 c^{2}\right)$. Thus $108 c^{6}=27 z_{0}^{2}\left[z_{0}^{2}-\right.$ $\left.\left(3+3 c^{2}\right)\right]^{2}=\left[\lambda+\left(2+3 c^{2}\right)\right]\left[\lambda-2\left(3+3 c^{2}\right)\right]^{2}$, which is equivalent to the equation (6).

Thus for fixed points $z_{1}, z_{2}, z_{3}$ of $P_{c}$, we have $\left|P_{c}^{\prime}\left(z_{1}\right)+P_{c}^{\prime}\left(z_{2}\right)+P_{c}^{\prime}\left(z_{3}\right)\right|=$ $\left|12+9 c^{2}\right|=\left|12+1 / 4+9\left(c^{2}-1 / 36\right)\right| \geq 12+1 / 4-9 / 36=12$. It follows that $\max \left\{\left|P_{c}^{\prime}\left(z_{1}\right)\right|,\left|P_{c}^{\prime}\left(z_{2}\right)\right|,\left|P_{c}^{\prime}\left(z_{3}\right)\right|\right\} \geq 4$. Thus we see that

$$
\max _{P(z)=z}\left\{\left|P^{\prime}(z)\right|\right\} \geq 4 .
$$

Finally suppose $P(z)=z^{4}+(-1 \pm 2 i) z$. It is easily seen that

$$
\max _{P(z)=z}\left\{\left|P^{\prime}(z)\right|\right\}=\sqrt{85}>9 .
$$

By (4), (5), (7) and (8), we see that (1) holds for $k=2$. Thus we have proved Lemma 9 . 
By Lemma 2 and Lemma 8 or Satz 1 in [4], we have the following result.

Lemma 10. Let $(f, U, V)$ be a polynomial-like mapping of degree $\geq 2$ and $k \geq 4$ be an integer. Then $f$ has at least one repelling periodic point of period $k$ in $U$.

Besides the above results coming from complex dynamics, we also require the following rescaling lemma due to Pang and Zalcman [18].

Lemma 11. ([18, Lemma 2]) Let $\mathcal{F}$ be a family of functions holomorphic on the unit disc, all of whose zeros have multiplicity at least $k$, and suppose that there exists $A \geq 1$ such that $\left|f^{(k)}(z)\right| \leq A$ whenever $f(z)=0$. Then if $\mathcal{F}$ is not normal, there exist, for each $0 \leq \alpha \leq k$,

a) a number $0<r<1$;

b) points $z_{n},\left|z_{n}\right|<r$;

c) functions $f_{n} \in \mathcal{F}$; and

d) positive numbers $\rho_{n} \rightarrow 0$

such that $\rho_{n}^{-\alpha} f_{n}\left(z_{n}+\rho_{n} \zeta\right)=: g_{n}(\zeta) \rightarrow g(\zeta)$ locally uniformly, where $g$ is a nonconstant entire function on $\mathbb{C}$, all of whose zeros have multiplicity at least $k$, such that the spherical derivative $g^{\#}(\zeta)$ of $g$ satisfies $g^{\#}(\zeta) \leq$ $g^{\#}(0)=k A+1$ for all $\zeta \in \mathbb{C}$.

We shall use the special case $\alpha=k=1$ of Lemma 11, which can be found in [17, Lemma 2].

Finally, we also need a result from graph theory, which is used implicitly in [13], [14] and stated formally in [3].

Let $V$ be a finite set and $E \subset V \times V$. Then the pair $G=(V, E)$ is called a digraph. An element of $V$ is called a vertex and an element of $E$ is called an edge. The edges are allowed to be of the form $(v, v)$ with $v \in V$, which is different from the usual terminology.

Let $k \in \mathbb{N}$ and $w=\left(v_{0}, \ldots, v_{k}\right) \in V^{k+1}$. We call $w$ a primitive closed walk of length $k$ in $G$ if $v_{0}=v_{k},\left(v_{j-1}, v_{j}\right) \in E$ for every $1 \leq j \leq k$, and there is no $p \in \mathbb{N}, 1 \leq p<k$, such that $p \mid k$ and $v_{j}=v_{l}$ for all $1 \leq j, l \leq k$ satisfying $p \mid(j-l)$.

Finally define the outdegree of a vertex $v$ to be the cardinality of the set $\{u \in V:(v, u) \in E\}$. 
Lemma 12. ([3, Lemma 9]) Let $q, n \in \mathbb{N}$ with $q \geq 6$ and $n \geq 2$, and let $G$ be a digraph with $q$ vertices such that the outdegree of each vertex is at least $q-2$. Then $G$ contains a primitive closed walk of length $n$.

\section{§3. Proofs of Theorems 1-3}

Proof of Theorem 1. Suppose that $\mathcal{F}$ is not quasinormal of order at most 1 . Then there exist $q \geq 2$ points $a_{j} \in D$ and a sequence $\left\{f_{n}\right\} \subset \mathcal{F}$ such that no subsequence of $\left\{f_{n}\right\}$ is normal in a neighborhood of any $a_{j}$. By Theorem B, we may assume that the integers $k\left(f_{n}\right)$ satisfy $k\left(f_{n}\right) \geq 3$.

Set $\delta<\frac{1}{2} \min \left\{\left|a_{i}-a_{j}\right|: i \neq j, 1 \leq i, j \leq q\right\}$ and $\varepsilon=\delta / \lambda$, where $\lambda=3 M \geq 3$. Bergweiler [7, Proof of Theorem 1.1] considered the digraph $G=(V, E)$ whose vertices are the $a_{j}$ and whose edges are all pairs $\left(a_{i}, a_{j}\right)$ for which $f_{n}$ has a simple island that is over $D\left(a_{j}, \delta\right)$ and contained in $D\left(a_{i}, \varepsilon\right)$. For the case $q \geq 6$, using the Ahlfors islands theorem (Lemma 1), he showed that the outdegree of each vertex is at least $q-2$. Hence, by Lemma $12, G$ contains a primitive closed walk $\left\{a_{i_{0}}, a_{i_{1}}, \ldots, a_{i_{k-1}}, a_{i_{k}}\right\}\left(a_{i_{k}}=a_{i_{0}}\right)$ of length $k=k\left(f_{n}\right) \geq 2$. Using the theory of polynomial-like mappings (Lemma 4), Bergweiler [7, Proof of Theorem 1.1] proved that the latter conclusion also holds when $\mathcal{F}$ is quasinormal of order $2 \leq q \leq 5$.

Hence $D\left(a_{i_{k-1}}, \varepsilon\right)$ contains a simple island $U_{k-1}$ over $D\left(a_{i_{k}}, \delta\right)=$ $D\left(a_{i_{0}}, \delta\right)$. Next $D\left(a_{i_{k-2}}, \varepsilon\right)$ contains a simple island $U_{k-2}$ over $D\left(a_{i_{k-1}}, \delta\right)$ and thus in particular, $D\left(a_{i_{k-2}}, \varepsilon\right)$ contains a simple island $U_{k-2}^{\prime}$ over $U_{k-1}$. Inductively, for $j=k-3, \ldots, 1,0, D\left(a_{i_{j}}, \varepsilon\right)$ contains a simple island $U_{j}$ over $D\left(a_{i_{j+1}}, \delta\right)$ and $D\left(a_{i_{j}}, \varepsilon\right)$ contains a simple island $U_{j}^{\prime}$ over $U_{j+1}^{\prime}$.

By Lemma 6, we see that $U_{k-1}^{\prime}=U_{k-1} \subset D\left(c_{k-1}, r_{k-1}\right)=D\left(a_{i_{k-1}}, \varepsilon\right)$ (say), and inductively, for $j=k-2, \ldots, 1,0$,

$$
U_{j}^{\prime}=f_{n}^{-1}\left(U_{j+1}^{\prime}\right) \subset f_{n}^{-1}\left(D\left(c_{j+1}, r_{j+1}\right)\right) \subset D\left(c_{j}, r_{j}\right) \subset D\left(a_{i_{j}}, \varepsilon\right),
$$

where

$$
r_{j}<\frac{3}{\lambda} r_{j+1}
$$

It follows that $U_{0}^{\prime} \subset D\left(c_{0}, r_{0}\right) \subset D\left(a_{i_{0}}, \varepsilon\right)$ and

$$
r_{0}<\left(\frac{3}{\lambda}\right)^{k-1} \varepsilon
$$

Thus by Lemma $5, f_{n}^{k}$ has a fixed point $\zeta_{0} \in U_{0}^{\prime} \subset D$ such that

$$
\left|\left(f_{n}^{k}\right)^{\prime}\left(\zeta_{0}\right)\right| \geq \frac{3}{4} \cdot \frac{\lambda \varepsilon}{r_{0}}>\frac{9}{4}\left(\frac{3}{\lambda}\right)^{k}>M^{k} .
$$


On the other hand, as the walk $\left\{a_{i_{0}}, a_{i_{1}}, \ldots, a_{i_{k-1}}, a_{i_{k}}\right\}$ is primitive, the fixed point $\zeta_{0}$ of $f_{n}^{k}$ is in fact a periodic point of period $k$ of $f_{n}$. This contradicts the condition that $f_{n} \in \mathcal{F}$. The proof of Theorem 1 is complete.

Proof of Theorem 2. Suppose that $\mathcal{F}$ is not normal at some $z_{0} \in D$. Then by Theorem 1 , there exists a sequence $\left\{f_{n}\right\} \subset \mathcal{F}$ which is normal in $D \backslash\left\{z_{0}\right\}$ such that no subsequence of $\left\{f_{n}\right\}$ is normal at $z_{0}$. By the maximum principle, we have $f_{n} \rightarrow \infty$ in $D \backslash\left\{z_{0}\right\}$.

Choose a positive number $\delta$ such that $\bar{D}\left(z_{0}, \delta\right) \subset D$, and let $R=\left(\left|z_{0}\right|+\right.$ $\delta) M$. Since $f_{n} \rightarrow \infty$ in $D \backslash\left\{z_{0}\right\}$, for sufficiently large $n,\left|f_{n}(z)\right|>R$ on $\left|z-z_{0}\right|=\delta$. However, as no subsequence of $\left\{f_{n}\right\}$ is normal at $z_{0}$, for sufficiently large $n,\left|f_{n}\left(z_{n}\right)\right|<R$ for some point $z_{n} \in \Delta\left(z_{0}, \delta\right)$, so that $f_{n}^{-1}(D(0, R))$ has a component $U_{n} \subset D\left(z_{0}, \delta\right)$. Note that $U_{n} \subset D(0, R / M)$. Thus the triple $\left(f_{n}, U_{n}, D(0, R)\right)$ is a polynomial-like mapping. By Lemma $3, f_{n}$ has at least one weakly repelling fixed point $z_{n} \in U_{n} \subset D$. This however contradicts the hypotheses. Theorem 2 is proved.

Proof of Theorem 3. First, we consider the case (c): $k(f) \geq 4$ for all $f \in \mathcal{F}$. Suppose $\mathcal{F}$ is not normal at some $z_{0} \in D$. Then by Theorem 1 or Theorem B, there exists a sequence $\left\{f_{n}\right\} \subset \mathcal{F}$ which is normal in the punctured domain $D \backslash\left\{z_{0}\right\}$ such that no subsequence of $\left\{f_{n}\right\}$ is normal at $z_{0}$. By the maximum principle, we have $f_{n} \rightarrow \infty$ in $D \backslash\left\{z_{0}\right\}$ as $n \rightarrow \infty$.

Choose a positive number $\delta$ such that $\bar{D}\left(z_{0}, \delta\right) \subset D$, and let $R>$ $4(K+1)\left(\left|z_{0}\right|+\delta\right)$. Since $f_{n} \rightarrow \infty$ in $D \backslash\left\{z_{0}\right\}$, for sufficiently large $n$, $\left|f_{n}(z)\right|>R$ on $\left|z-z_{0}\right|=\delta$. However, as no subsequence of $\left\{f_{n}\right\}$ is normal at $z_{0}$, for sufficiently large $n,\left|f_{n}\left(z_{n}\right)\right|<R$ for some point $z_{n} \in$ $D\left(z_{0}, \delta\right)$, so that $f_{n}^{-1}(D(0, R))$ has a component $U_{n} \subset D\left(z_{0}, \delta\right)$. Note that $U_{n} \subset D(0, R /[4(K+1)])$. Thus the triple $\left(f_{n}, U_{n}, D(0, R)\right)$ is a polynomiallike mapping of some degree $d \geq 1$. By Lemma 10 and the assumption that $f_{n}$ has no repelling periodic point of some period $k=k\left(f_{n}\right) \geq 4$, we see that $d=1$, so that $\left(f_{n}, U_{n}, D(0, R)\right)$ is a regular map. Since $R /[4(K+1)]<R / 2$, by Lemma 5 , we find that $f_{n}$ has a fixed point $\zeta \in U_{n} \subset D$ with $\left|f_{n}^{\prime}(\zeta)\right| \geq K+1>K$. This is a contradiction since we assumed every $f \in \mathcal{F}$ has no fixed point $z$ with $\left|f^{\prime}(z)\right|>K$. Thus we complete the proof of Theorem 3 for the case (c).

Now we consider the case (b): $K<2 \sqrt{2}+1$ and $k(f) \geq 3$ for all $f \in \mathcal{F}$. It follows from the case (c) that we may assume that $k(f)=3$ for all $f \in \mathcal{F}$.

Set

$$
\mathcal{G}=\{g=f-i d: f \in \mathcal{F}\}
$$


where $i d$ denotes the identity function. Since $\left|f^{\prime}(z)\right| \leq K$ whenever $f(z)=z$ for every $f \in \mathcal{F}$, we see that $\left|g^{\prime}(z)\right| \leq K+1$ whenever $g(z)=0$ for every $g \in \mathcal{G}$.

Obviously, $\mathcal{F}$ is normal in $D$ if and only if $\mathcal{G}$ is normal in $D$. Now suppose that $\mathcal{G}$ is not normal at $z_{0} \in D$. Then by Lemma 11, we can find points $z_{n} \rightarrow z_{0}$, positive numbers $\rho_{n} \rightarrow 0$ and functions $g_{n}=f_{n}-i d \in \mathcal{G}$ such that

$$
G_{n}(\zeta)=\frac{g_{n}\left(z_{n}+\rho_{n} \zeta\right)}{\rho_{n}} \rightarrow G(\zeta)
$$

locally uniformly on $\mathbb{C}$, where $G$ is a nonconstant entire function on $\mathbb{C}$ such that $G^{\#}(\zeta) \leq G^{\#}(0)=K+2$.

Set

$$
\begin{aligned}
M_{n}(\zeta) & =z_{n}+\rho_{n} \zeta \\
H_{n}(\zeta) & =G_{n}(\zeta)+\zeta
\end{aligned}
$$

Then by (12)-(14), we have

$$
H_{n}(\zeta)=\frac{f_{n}\left(M_{n}(\zeta)\right)-z_{n}}{\rho_{n}} .
$$

This with (13) yields $M_{n}\left(H_{n}(\zeta)\right)=z_{n}+\rho_{n} H_{n}(\zeta)=f_{n}\left(M_{n}(\zeta)\right)$. Thus $M_{n}$ conjugates $f_{n}$ and $H_{n}$, and hence $H_{n}^{j}=M_{n}^{-1} \circ f_{n}^{j} \circ M_{n}$ for every $j \in \mathbb{N}$. Thus we have

$$
f_{n}^{j}\left(M_{n}(\zeta)\right)=z_{n}+\rho_{n} H_{n}^{j}(\zeta), \quad j=1,2,3,
$$

and

$$
H_{n}^{j}(\zeta)-\zeta=\frac{f_{n}^{j}\left(M_{n}(\zeta)\right)-M_{n}(\zeta)}{\rho_{n}}, \quad j=1,2,3
$$

Let

$$
H(\zeta)=G(\zeta)+\zeta
$$

Then by (12) and (14), for $j=1,2,3$,

$$
H_{n}^{j}(\zeta) \rightarrow H^{j}(\zeta)
$$

locally uniformly on $\mathbb{C}$ as $n \rightarrow \infty$. 
If $H(\zeta)$ has a repelling periodic point $\zeta_{0} \in \mathbb{C}$ of period 3 , then there exist positive numbers $\lambda(>1), \delta$ and $\varepsilon$ such that $\left|\left(H^{3}\right)^{\prime}(\zeta)\right| \geq \lambda$ and $\left|H^{j}(\zeta)-\zeta\right| \geq \varepsilon,(j=1,2)$ for $\zeta \in \bar{D}\left(\zeta_{0}, \delta\right) \subset \mathbb{C}$. Thus by (19) and Hurwitz's Theorem, there exist points $\zeta_{n} \rightarrow \zeta_{0}$ such that $H_{n}^{3}\left(\zeta_{n}\right)=\zeta_{n},\left|\left(H_{n}^{3}\right)^{\prime}\left(\zeta_{n}\right)\right| \geq$ $(\lambda+1) / 2>1$ and $\left|H_{n}^{j}(\zeta)-\zeta\right| \geq \varepsilon / 2,(j=1,2)$ for $\zeta \in \bar{D}\left(\zeta_{n}, \delta / 2\right)$. Hence by (17), for sufficiently large $n, M_{n}\left(\zeta_{n}\right)$ is a repelling periodic point $\zeta_{0} \in \mathbb{C}$ of period 3 of $f_{n}$ in $D$. This contradicts the condition.

Hence $H(\zeta)$ has no repelling periodic point of period 3 in $\mathbb{C}$. Thus by Lemma 7 , we deduce that $H$ is a polynomial.

Similarly, we can find that $H$ has no fixed point $\zeta$ with $\left|H^{\prime}(\zeta)\right|>K$ in $\mathbb{C}$.

If $\operatorname{deg}(H) \geq 2$, then we see by Lemma 9 that $H$ has a fixed point $z_{0}$ such that $\left|H^{\prime}\left(z_{0}\right)\right| \geq 2 \sqrt{2}+1>K$. This is a contradiction.

Hence $\operatorname{deg}(H) \leq 1$, so that $H(\zeta)=A \zeta+B$ for some constants $A$ and $B$. Since $H(\zeta)$ has no fixed point $\zeta$ with $\left|H^{\prime}(\zeta)\right| \geq K$, we have that $|A| \leq K$. Hence $\left|G^{\prime}(\zeta)\right|=|A-1| \leq K+1$, which contradicts $G^{\#}(0)=K+2$.

Hence $\mathcal{G}$ is normal in $D$, so that $\mathcal{F}$ is normal in $D$. Thus the proof of the case (b) is complete.

Finally, we consider the case $(\mathrm{a}): K<3$ and $k(f) \geq 2$ for all $f \in \mathcal{F}$. The proof of this case is analogous to that of the case (b). We omit the details.

Remark 1. The reviewer pointed out in the report that the cases (a) and (b) can also be proved by using the theory of polynomial-like mappings. Indeed, the modulus of the annulus given by the difference between the range and the domain of the polynomial-like map can be chosen arbitrarily large. Therefore the dilatation of the quasiconformal conjugacy between this polynomial-like mapping and an actual polynomial can be arbitrarily small and hence the multipliers of the fixed points will be changed only by an arbitrarily small amount.

\section{§4. Examples}

Here we construct two examples mentioned in the first section. These examples are modifications of the extremal polynomials in Lemma 8. For instance, in Example 2, we first choose a polynomial $f(z)=z+z^{2}-1$ which has no periodic point of period 2 and the multipliers of whose fixed points are 3 and -1 respectively. Then the functions $\frac{1}{n} f(n z)=z+n\left(z^{2}-\frac{1}{n^{2}}\right)$ have the same property. Finally, we choose a suitable function $U_{n}$ which 
converges to 1 locally uniformly on the plane such that the functions $f_{n}(z)=$ $z+n\left(z^{2}-\frac{1}{n^{2}}\right) U_{n}(z)$ have no periodic points of period 2 and no fixed points in the unit disk $\Delta$ other than $\pm 1 / n$. In particular, the multipliers of the fixed points $\pm 1 / n$ are less than 3 .

\section{EXAMPLE 2. Let for every $n \in \mathbb{N}$}

$$
f_{n}(z)=z+n\left(z-\frac{1}{n}\right)\left(z+\frac{1}{n}\right) \frac{n^{4}-n z^{2}}{n^{4}+z} .
$$

Then every $f_{n}$ is holomorphic in the unit disk $\Delta=\{z:|z|<1\}$. By a direct computation, we have

$$
f_{n}^{2}(z)=z+n^{4}\left(z-\frac{1}{n}\right)\left(z+\frac{1}{n}\right)^{3} \frac{\left(n^{3}-z^{2}\right) P_{n}(z)}{\left(n^{4}+z\right)^{3} Q_{n}(z)},
$$

where

$$
\begin{aligned}
P_{n}(z)= & n^{6} z^{10}-2 n^{5} z^{9}+\left(-3 n^{9}-4 n^{4}\right) z^{8}+\left(2 n^{8}+10 n^{3}\right) z^{7} \\
& +\left(3 n^{12}+16 n^{7}+n^{2}\right) z^{6}+\left(2 n^{11}-14 n^{6}-12 n\right) z^{5} \\
& +\left(-n^{15}-14 n^{10}-20 n^{5}+6\right) z^{4}+\left(-2 n^{14}-4 n^{9}+22 n^{4}\right) z^{3} \\
& +\left(n^{13}+20 n^{8}-2 n^{3}\right) z^{2}+\left(6 n^{12}-4 n^{7}\right) z-n^{11}+n^{16} \\
= & n^{16}-z^{4} n^{15}-2 z^{3} n^{14}+z^{2} n^{13}+\left(3 z^{6}+6 z\right) n^{12}+\left(2 z^{5}-1\right) n^{11} \\
& -14 z^{4} n^{10}+\left(-3 z^{8}-4 z^{3}\right) n^{9}+\left(2 z^{7}+20 z^{2}\right) n^{8}+\left(16 z^{6}-4 z\right) n^{7} \\
& +\left(z^{10}-14 z^{5}\right) n^{6}+\left(-2 z^{9}-20 z^{4}\right) n^{5}+\left(-4 z^{8}+22 z^{3}\right) n^{4} \\
& +\left(10 z^{7}-2 z^{2}\right) n^{3}+z^{6} n^{2}-12 z^{5} n+6 z^{4}, \\
(23) \quad Q_{n}(z)=-n^{2} z^{4}+\left(n^{5}+2\right) z^{2}+2 n^{4} z+n^{8}-n^{3} & =n^{8}+z^{2} n^{5}+2 z n^{4}-n^{3}-z^{4} n^{2}+2 z^{2} .
\end{aligned}
$$

Thus we see that $f_{n}$ has no periodic point of period 2 in $\Delta$ for $n \geq 179$. Indeed, suppose that $f_{n}$ has a periodic point $z_{0}$ of period 2 in $\Delta$. Then $\left|z_{0}\right|<1$, and by $(20)-(23)$, we have $P_{n}\left(z_{0}\right)=0$, so that

$$
\begin{aligned}
n^{16}= & \mid-z_{0}^{4} n^{15}-2 z_{0}^{3} n^{14}+z_{0}^{2} n^{13}+\left(3 z_{0}^{6}+6 z_{0}\right) n^{12}+\left(2 z_{0}^{5}-1\right) n^{11} \\
& -14 z_{0}^{4} n^{10}-\left(3 z_{0}^{8}+4 z_{0}^{3}\right) n^{9}+\left(2 z_{0}^{7}+20 z_{0}^{2}\right) n^{8}+\left(16 z_{0}^{6}-4 z\right) n^{7} \\
& +\left(z_{0}^{10}-14 z_{0}^{5}\right) n^{6}-\left(2 z_{0}^{9}+20 z_{0}^{4}\right) n^{5}+\left(-4 z_{0}^{8}+22 z_{0}^{3}\right) n^{4} \\
& +\left(10 z_{0}^{7}-2 z_{0}^{2}\right) n^{3}+z_{0}^{6} n^{2}-12 z_{0}^{5} n+6 z_{0}^{4} \mid
\end{aligned}
$$




$$
\begin{aligned}
\leq & n^{15}+2 n^{14}+n^{13}+9 n^{12}+3 n^{11}+14 n^{10}+7 n^{9}+27 n^{8} \\
& +20 n^{7}+15 n^{6}+22 n^{5}+26 n^{4}+12 n^{3}+n^{2}+12 n+6 \\
\leq & 178 n^{15}
\end{aligned}
$$

It follows that $n \leq 178$.

We also can see that every $f_{n} \in \mathcal{F}$ has two fixed points $z=1 / n$ and $z=-1 / n$ with multiplier

$$
f_{n}^{\prime}(1 / n)=3-\frac{4}{n^{5}+1}, \quad \text { and } \quad f_{n}^{\prime}(-1 / n)=-1,
$$

so that $\left|f_{n}^{\prime}(z)\right|<3$ whenever $f_{n}(z)=z$ in $\Delta$.

The family $\left\{f_{n}: n \geq 179\right\}$ is not equicontinuous since $f_{n}(1 / n) \rightarrow 0$ and $f_{n}(1 / \sqrt{n}) \rightarrow 1$ as $n \rightarrow \infty$. It follows that the family $\left\{f_{n}: n \geq 179\right\}$ is not normal in the unit disk $\Delta$.

EXAMPLE 3. Let for every $n \in \mathbb{N}$

$$
f_{n}(z)=z+\frac{1}{n}\left(n^{2} z^{2}-2\right)\left(1-\frac{\left(n^{3} z^{3}+2 n^{2} z^{2}-n z-1\right)^{2}}{n^{32}}\right) .
$$

Then every $f_{n}$ is holomorphic in the unit disk $\Delta=\{z:|z|<1\}$.

Obviously, for $n \geq 2$, every $f_{n}$ has no fixed point in $\Delta$ except $\pm \sqrt{2} / n$. Next we claim that there exists an integer $n_{0} \geq 2$ such that for $n \geq n_{0}$, every $f_{n}$ has no periodic point of period 3 in $\Delta$ except $z_{1} / n, z_{2} / n, z_{3} / n$, where $z_{j}(j=1,2,3)$ are roots of the equation $z^{3}+2 z^{2}-z-1=0$.

Let $\zeta \notin\left\{z_{1} / n, z_{2} / n, z_{3} / n\right\}$ be a periodic point of period 3 of $f_{n}$ in $\Delta$. Then $\zeta, f_{n}(\zeta), f_{n}^{2}(\zeta) \in \Delta$ and $f_{n}^{3}(\zeta)=\zeta$. Since $f_{n}^{3}(z)$ is a polynomial, whose coefficients are rational functions in $n$, it follows from $f_{n}^{3}(\zeta)=\zeta$ and $\zeta \in \Delta$ that

$$
\zeta=c_{0}+\frac{c_{1}}{n^{s_{1}}}+o\left(\frac{1}{n^{s_{1}}}\right)
$$

as $n \rightarrow \infty$, where $c_{0}, c_{1}, s_{1}$ are constants not depending on $n$ and satisfying $\left|c_{0}\right| \leq 1$ and $s_{1}>0$.

First we show that $c_{0}=0$. If it is not the case, then by (24) and (25), we have $f_{n}(\zeta)=c_{0}^{2} n+o(n)$. This contradicts $f_{n}(\zeta) \in \Delta$. Thus $c_{0}=0$.

Next we prove $s_{1}=1$. In fact, if $s_{1}>1$, then by (24) and (25), we have $f_{n}(\zeta)=-2 / n+o(1 / n)$ and then $f_{n}^{2}(\zeta)=o(1 / n)$, so that $\zeta=f_{n}^{3}(\zeta)=$ $-2 / n+o(1 / n)$. This contradicts $(25)$ as $c_{0}=0$ and $s_{1}>1$. A similar 
argument yields that $s_{1}<1$ is also impossible. Thus we have proved that $s_{1}=1$. Hence

$$
\zeta=\frac{c_{1}}{n}+o\left(\frac{1}{n}\right) .
$$

Final, we prove that $c_{1} \in\left\{z_{1}, z_{2}, z_{3}\right\}$ and $o(1 / n) \equiv 0$ exactly in (26). For otherwise, we must have

$$
\zeta=\frac{c_{1}}{n}+\frac{c_{2}}{n^{s_{2}}}+o\left(\frac{1}{n^{s_{2}}}\right),
$$

where $c_{2} \neq 0$ and $s_{2}>1$ are constants. Thus by (24), we have

$$
\begin{aligned}
& f_{n}^{3}(\zeta)=\frac{\left(c_{1}\right)^{8}+4\left(c_{1}\right)^{7}-14\left(c_{1}\right)^{5}-7\left(c_{1}\right)^{4}+14\left(c_{1}\right)^{3}+7\left(c_{1}\right)^{2}-3 c_{1}-2}{n} \\
& +\frac{\left[8\left(c_{1}\right)^{7}+28\left(c_{1}\right)^{6}+4\left(c_{1}\right)^{5}-60\left(c_{1}\right)^{4}-32\left(c_{1}\right)^{3}+26\left(c_{1}\right)^{2}+10 c_{1}-2\right] c_{2}}{n^{s_{2}}} \\
& \quad+o\left(\frac{1}{n^{s_{2}}}\right) .
\end{aligned}
$$

$$
\begin{aligned}
f_{n}(\zeta) & =\frac{\left(c_{1}\right)^{2}+c_{1}-2}{n}+\frac{2 c_{1} c_{2}+c_{2}}{n^{s_{2}}}+o\left(\frac{1}{n^{s_{2}}}\right) . \\
f_{n}^{2}(\zeta)= & \frac{\left(c_{1}\right)^{4}+2\left(c_{1}\right)^{3}-2\left(c_{1}\right)^{2}-3 c_{1}}{n} \\
& +\frac{\left[4\left(c_{1}\right)^{3}+6\left(c_{1}\right)^{2}-2 c_{1}-2\right] c_{2}}{n^{s_{2}}}+o\left(\frac{1}{n^{s_{2}}}\right) .
\end{aligned}
$$

Since $\zeta=f_{n}^{3}(\zeta)$, we get by $(26)$ and (30) that

(31) $\left(c_{1}\right)^{8}+4\left(c_{1}\right)^{7}-14\left(c_{1}\right)^{5}-7\left(c_{1}\right)^{4}+14\left(c_{1}\right)^{3}+7\left(c_{1}\right)^{2}-3 c_{1}-2=c_{1}$,

(32) $\left[8\left(c_{1}\right)^{7}+28\left(c_{1}\right)^{6}+4\left(c_{1}\right)^{5}-60\left(c_{1}\right)^{4}-32\left(c_{1}\right)^{3}+26\left(c_{1}\right)^{2}\right.$

$$
\left.+10 c_{1}-2\right] c_{2}=c_{2} \text {. }
$$

By (31), we can see that either $\left(c_{1}\right)^{2}=2$ or $\left(c_{1}\right)^{3}+2\left(c_{1}\right)^{2}-c_{1}-1=0$. Each case with (32) shows that $c_{2}=0$. Thus

$$
\zeta=\frac{c_{1}}{n}
$$

If $\left(c_{1}\right)^{2}=2$, then $\zeta$ is a fixed point of $f_{n}$, contradicting that $\zeta$ is a periodic point of period 3. Thus $\left(c_{1}\right)^{3}+2\left(c_{1}\right)^{2}-c_{1}-1=0$. That is $c_{1} \in\left\{z_{1}, z_{2}, z_{3}\right\}$. 
Thus we have proved the above claim. Now consider the multipliers of the fixed points $\pm \sqrt{2} / n$ and periodic points $z_{1} / n, z_{2} / n, z_{3} / n$ of period 3 of $f_{n}$ in $\Delta$. By computation, we have

$$
\begin{gathered}
f_{n}^{\prime}(\sqrt{2} / n)=2 \sqrt{2}+1-\frac{24+22 \sqrt{2}}{n^{32}}, \\
f_{n}^{\prime}(-\sqrt{2} / n)=-2 \sqrt{2}+1-\frac{24-22 \sqrt{2}}{n^{32}},
\end{gathered}
$$

so that for $n \geq 2,\left|f_{n}^{\prime}(z)\right|<2 \sqrt{2}+1$ whenever $f_{n}(z)=z$ in $\Delta$.

We also can see that $z_{1} / n, z_{2} / n, z_{3} / n$ are periodic points of period 3 of $f_{n}$ with multiplier 1 .

But one can see that the family $\mathcal{F}=\left\{f_{n}(z): n \geq n_{0}\right\}$ is not normal at the origin.

Remark. Following [8], we say $P$ is a Bloch property if the following two assertions

(a) If $\langle f, \mathbb{C}\rangle \in P$, then $f$ is constant, and

(b) The family $\{f:\langle f, D\rangle \in P\}$ is normal on $D$ for each domain $D \subset \mathbb{C}$

are equivalent. A Bloch property such that both (a) and (b) are true is called a Picard-Montel property.

From Examples 2 and 3, we see that there exist Bloch properties $P$ that are not Picard-Montel properties such that the assertion

$\left(\mathrm{a}^{\prime}\right)$ The family $\{f:\langle f, \mathbb{C}\rangle \in P\}$ is normal on $\mathbb{C}$

is true.

Indeed, using Lemma 7 and Lemma 9 in the present paper, one can verify that the property $P$ :

$f$ has no repelling periodic point of period 2 and $f$ has no fixed point $\zeta$ satisfying $\left|f^{\prime}(\zeta)\right| \geq 3$,

and the property $Q$ :

$f$ has no repelling periodic point of period 3 and $f$ has no fixed point $\zeta$ satisfying $\left|f^{\prime}(\zeta)\right| \geq 2 \sqrt{2}+1$

are the desired properties. 


\section{§5. A remark on families of meromorphic functions}

Using a similar argument as in the first part of the proof of Theorem 1, we have the following result.

TheOREM 4. Let $M \geq 1$ be a positive number, $D \subset \mathbb{C}$ be a domain, and $\mathcal{F}$ be a family of all meromorphic functions $f$ in $D$ for which there exists an integer $k=k(f) \geq 2$ such that $\left|\left(f^{k}\right)^{\prime}(\zeta)\right| \leq M^{k}$ for all periodic points $\zeta$ of period $k$ of $f$ in $D$, then $\mathcal{F}$ is quasinormal of finite order.

However, we do not know the exact order of the quasi-normality. The following example shows that the order of the quasi-normality is at least 2 . It seems likely that the number 2 is the exact order of the quasi-normality.

EXAMPLE 4. Let

$$
\mathcal{F}=\left\{f_{n}(z)=\frac{1}{n z(z-1)}: n \in \mathbb{N}\right\}
$$

Then $\mathcal{F}$ is a family of functions meromorphic in $\mathbb{C}$. And for every $f_{n} \in \mathcal{F}$, we have

$$
f_{n}(z)=z-\frac{n z^{3}-n z^{2}-1}{n z(z-1)}, \quad \text { and } \quad f_{n}^{2}(z)=z-\frac{z\left(n z^{3}-n z^{2}-1\right)}{n z^{2}-n z-1} .
$$

Thus every $f_{n} \in \mathcal{F}$ has no periodic point of period 2 in $\mathbb{C}$. On the other hand, it is easy to check that $\mathcal{F}$ is quasinormal of order 2 in $\mathbb{C}$.

Acknowledgement. I am extremely grateful to the referee for his or her many valuable suggestions which improve my paper both on the contents and the readability.

\section{REFERENCES}

[1] I. N. Baker, Fix-points of polynomials and rational functions, J. London Math. Soc. (2), 39 (1964), 615-622.

[2] I. N. Baker, Repulsive fixpoints of entire functions, Math. Z., 104 (1968), 252-256.

[3] D. Bargmann and W. Bergweiler, Periodic points and normal families, Proc. Amer. Math. Soc., 129 (2001), 2881-2888.

[4] W. Bergweiler, Periodische Punkte bei der Iteration ganzer Funktionen, Habilitationsschrift, Rheinisch-Westfälische Techn. Hochsch., Aachen, 1991.

[5] W. Bergweiler, Periodic points of entire functions: proof of a conjecture of Baker, Complex Variables Theory Appl., 17 (1991), 57-72. 
[6] W. Bergweiler, A new proof of the Ahlfors five islands theorem, J. Analyse Math., 76 (1998), 337-347.

[7] W. Bergweiler, Quasinormal families and periodic points, Contemp. Math., 382 (2005), 55-63.

[8] W. Bergweiler, Bloch's principle, Comput. Methods Funct. Theory, 6 (2006), 77-108.

[9] L. Carleson and T. W. Gamelin, Complex Dynamics, Springer, New York, Berlin, Heidelberg, 1993.

[10] J. M. Chang, Polynomials without repelling periodic points of given period, J. Math. Anal. Appl. (1), 324 (2006), 1-13.

[11] J. M. Chang and M. L. Fang, Normal families and fixed points, J. Analyse Math., 95 (2005), 389-395.

[12] A. Douady and J. H. Hubbard, On the dynamics of polynomial-like mappings, Ann. Sci. École Norm. Sup. (4), 18 (1985), 287-343.

[13] M. Essén and S. J. Wu, Fixpoints and normal families of analytic functions, Complex Variables, 37 (1998), 171-178.

[14] M. Essén and S. J. Wu, Repulsive fixpoints of analytic functions with application to complex dynamics, J. London Math. Soc. (2), 62 (2000), 139-148.

[15] W. K. Hayman, Meromorphic Functions, Clarendon Press, Oxford, 1964.

[16] W. K. Hayman, Research problems in function theory, Athlone Press, London, 1967.

[17] X. C. Pang, Shared values and normal families, Analysis, 22 (2002), 175-182.

[18] X. C. Pang and L. Zalcman, Normal families and shared values, Bull. London Math. Soc., 32 (2000), 325-331.

[19] J. Schiff, Normal Families, Springer-Verlag, 1993.

[20] L. Yang, Some recent results and problems in the theory of value distribution, Proc. of the Symposium on Value Distribution Theory in Several Complex Variables (W. Stoll, ed.), University of Notre Dame Press, 1992, pp. 157-171.

[21] L. Yang, Value Distribution Theory, Springer-Verlag, Berlin, 1993.

Department of Mathematics

Changshu Institute of Technology

Changshu, Jiangsu 215500

P. R. China

jmchang@cslg.edu.cn 